\title{
One-step fabrication of silver nanoparticle embedded polymer nanofibers by radical-mediated dispersion polymerization
}

\author{
Hyeyoung Kong and Jyongsik Jang* \\ Received (in Cambridge, UK) 12th April 2006, Accepted 25th May 2006 \\ First published as an Advance Article on the web 13th June 2006 \\ DOI: $10.1039 / \mathrm{b} 605286 \mathrm{j}$
}

Silver nanoparticle embedded poly(vinyl alcohol)-poly(methyl methacrylate) nanofibers, $c a .30 \mathrm{~nm}$ in diameter and $c a .60 \mu \mathrm{m}$ in length, were fabricated by one-step radical-mediated dispersion polymerization using $2,2^{\prime}$-azobis(isobutyronitrile) to reduce the silver ions. In this methodology, PVA acted both as a gelator to form the nanofibers and as a stabilizer to protect the silver clusters from sintering.

Silver-polymer nanostructures have been extensively studied because of their potential applications, ranging from electronic and optical devices to biosensing and antimicrobial agents. ${ }^{1}$ The dichroic behaviors of silver-polymer nanostructures have been investigated and applied in bicolored liquid crystal displays. ${ }^{1}$ In general, there are two synthetic approaches of silver-polymer nanostructures: in situ and ex situ methods. ${ }^{2}$ The ex situ method involves silver nanoparticle formation first, followed by dispersion into a polymer matrix. ${ }^{3}$ In the in situ approach, metal nanoparticles can be generated inside a polymer by reduction of metallic precursor which is dissolved in the polymer or the polymerizing solution. ${ }^{4}$

Recently, Chujo et al. synthesized silver dendritic structures by a redox reaction between a $\pi$-conjugated polymer and silver ions in solution. ${ }^{5}$ They showed that a $\pi$-conjugated polymer containing a strong electron-donating unit could reduce silver ions. $\mathrm{Yu}$ and coworkers fabricated flexible silver-crosslinked poly(vinyl alcohol) (PVA) coaxial nanocables by in situ reduction of silver ions and crosslinking of PVA. ${ }^{6}$ They explained that PVA was responsible for both the formation of silver nanoparticles and growth of silver nanowires in an axial direction. In addition to these in situ metalizations in polymeric solutions, a monomer and a radical initiator have been also used to form silver nanostructures during polymerization. Chemical oxidative polymerization of a pyrrole monomer was performed by reducing silver ions in a redox system. ${ }^{7}$ Moreover, in situ formation of silver nanoparticles occurred onto the polymer microsphere surface due to the reduction potential of the generated radicals. ${ }^{8}$

Silver-polymer nanofibers have been applied to various research fields such as biomaterials, medical devices, and electronics. ${ }^{9}$ Most of these polymer nanofibers have been prepared by the electrospinning technique, which needs a supplementary electric potential to create a jet for the formation of fibers. In the preparation of silver-polymer nanofibers, silver nanoparticles can be deposited on those electrospun polymer nanofibers by sputter coating, ${ }^{9}$ or

Hyperstructured Organic Materials Research Center and School of Chemical and Biological Engineering, College of Engineering, Seoul National University, Shinlimdong 56-1, Seoul, 151-742, Korea.

E-mail: jsjang@plaza.snu.ac.kr; Fax: +82 2888 1604; Tel: +82 28807069 polymeric solutions containing silver ions can be co-electrospun. ${ }^{10}$ Therefore, facile synthesis of silver-polymer nanofibers using in situ methods is still challenging and of particular interest. In this communication, we report the novel one-step fabrication of silver nanoparticle embedded poly(vinyl alcohol)-poly(methyl methacrylate) (PVA-PMMA) nanofibers by radical-mediated dispersion polymerization. In order to synthesize silver-polymer nanofibers by one-step polymerization, we selected 2,2'-azobis(isobutyronitrile) (AIBN), which played a role in initiating the radical polymerization, as well as reducing silver ions.

Scheme 1 illustrates the overall synthetic procedure for the fabrication of silver nanoparticle embedded polymer nanofibers. PVA has a lone pair on the hydroxyl oxygen, which can coordinate with metal ions. To form silver ion-PVA complexes, the PVA was introduced into aqueous silver nitrate solution $(0.29 \mu \mathrm{M})$. Different amounts $\left(0.25-2.0 \mathrm{~g} \mathrm{~L}^{-1}\right)$ of PVA (from Sigma-Aldrich, with molecular weight in the range 31000 181000 ) were used. Vigorous magnetic stirring was used to induce a high shear flow during the fabrication of the polymer nanofibers. Under these conditions, PVA became fully oriented with silver ions in the flow direction. ${ }^{11}$ AIBN $(0.29 \mu \mathrm{M})$, as a reductant and radical initiator, was then added into the oriented silver ion-PVA aqueous solution $(100 \mathrm{~mL})$ at $60{ }^{\circ} \mathrm{C}$. The organic radicals produced by the decomposition of AIBN were adsorbed onto the surfaces of the silver ion-PVA complexes, which were relatively hydrophobic compared to the hydrophilic aqueous medium. Simultaneously, the adsorbed radicals reduced silver ions to form linear silver nanoparticles-PVA assemblies, due to the dipole-dipole interaction between the silver nanoaprticles. ${ }^{12}$ At this stage, PVA acted as not only a gelator ${ }^{13}$ but also a stabilizing agent to prohibit silver clusters sintering. When MMA (0.02 M) was injected, the

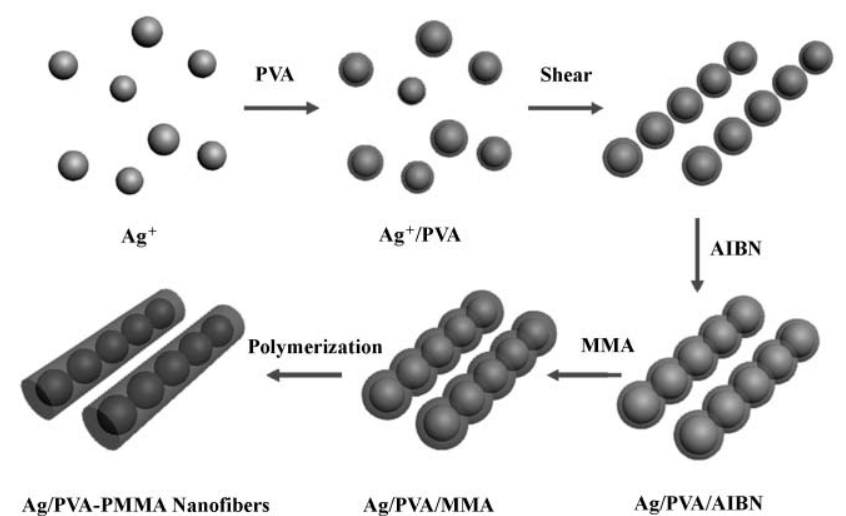

Scheme 1 Schematic illustration of the synthesis of silver nanoparticle embedded polymer nanofibers. 
remaining radicals initiated the polymerization of MMA monomer, spontaneously reducing the silver ions. The injected MMA was adsorbed onto the linear silver-PVA assemblies because hydrogen bonds were formed between the hydroxyl $(-\mathrm{OH})$ functional group of PVA and the carboxyl (-COO) group of MMA. As a result, MMA became tightly bound to PVA, and then polymerized onto the silver-PVA complex. Polymerization was allowed to proceed for $24 \mathrm{~h}$, and the products precipitated during this time. The precipitate was collected and washed with ethanol to remove the residual reagents.

Fig. 1a shows scanning electron microscopy (SEM) and transmission electron microscopy (TEM, inset) images of silver nanoparticle embedded polymer nanofibers. The polymer nanofibers were $c a .30 \mathrm{~nm}$ in diameter and $c a .60 \mu \mathrm{m}$ in length, as can be seen in the SEM image. Furthermore, they had smooth surfaces, indicating that no silver nanoparticles were located on the surface of the nanofibers. The inset TEM image in Fig. 1a shows that silver nanoparticles with an average size of $7 \mathrm{~nm}$ were embedded in the polymer nanofibers and assembled in one direction. Judging from these data, silver nanoparticle-polymer nanofibers have been formed by this method.

In addition, the optical properties of the silver-polymer nanofibers were analyzed by absorption spectroscopy. The plasmon absorption peak at $400 \mathrm{~nm}$ is characteristic of nanosize

(a)

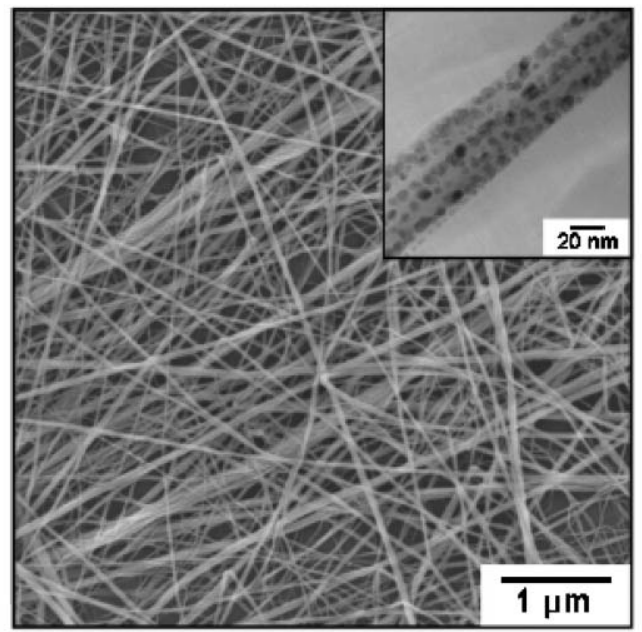

(b)

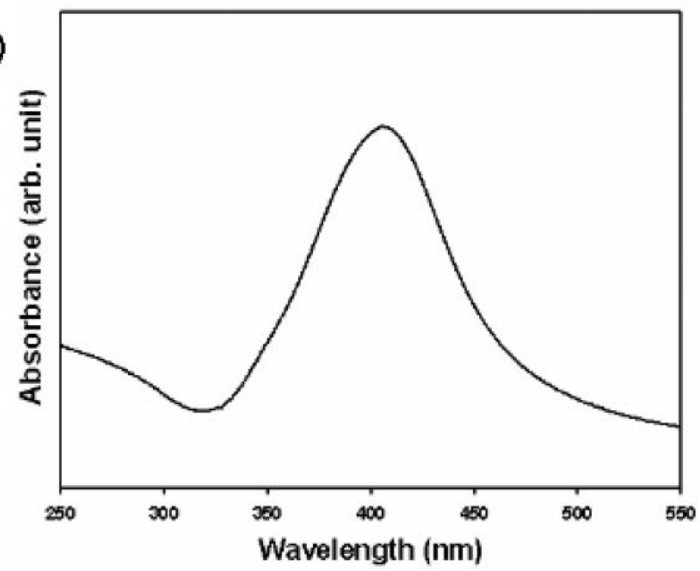

Fig. 1 (a) SEM and TEM (inset) photographs, and (b) UV-Vis absorption spectrum of silver nanoparticle embedded polymer nanofibers. silver particles, and broadness of this peak is indicative of the small size of these nanoparticles. ${ }^{14}$ Fig. $1 \mathrm{~b}$ displays the broad characteristic peak of silver nanoparticles in the UV-Vis spectrum. This obervation confirmed that the silver nanoparticles were nanometre-sized. By using the correlation between the full width at half-maximum (FWHM) and the diameter of particles, ${ }^{14}$ the size of the silver nanoparticles embedded in the polymer matrix was determined as approximately $8 \mathrm{~nm}$. This result is consistent with the TEM image.

An X-ray photoelectron spectrum (XPS) of the prepared silver nanoparticles is presented in Fig. 2a. Generally, metallic silver 3d peaks are centered at $367.9 \mathrm{eV}$ (for $\mathrm{Ag} 3 \mathrm{~d}_{5 / 2}$ ) and $373.9 \mathrm{eV}$ (for $\mathrm{Ag}$ $3 \mathrm{~d}_{3 / 2}$ ), with a spin energy separation of $6.0 \mathrm{eV} .^{15}$ Under our experimental conditions, two peaks corresponding to the embedded silver nanoparticles were observed at $368 \mathrm{eV}$ and $374 \mathrm{eV}$, with a $6.0 \mathrm{eV}$ separation, corresponding to the $\mathrm{Ag} 3 \mathrm{~d}_{5 / 2}$ and $\mathrm{Ag} 3 \mathrm{~d}_{3 / 2}$ binding energies, respectively. Our prepared silver nanoparticles therefore had negligible shifts and the same spin energy separation as metallic silver, thus implying that the silver nanoparticles synthesized during polymerization are mostly zerovalent silver. A Fourier-transform infrared (FT-IR) spectroscopic analysis was conducted to confirm the polymerization at the surface of silver nanoparticles (Fig. 2b). In the FT-IR spectrum of Ag-PVA, the peaks from PVA could be assigned: 1150 and $1195 \mathrm{~cm}^{-1}$ from the $\mathrm{C}-\mathrm{O}$ stretching vibration, and near $3400 \mathrm{~cm}^{-1}$
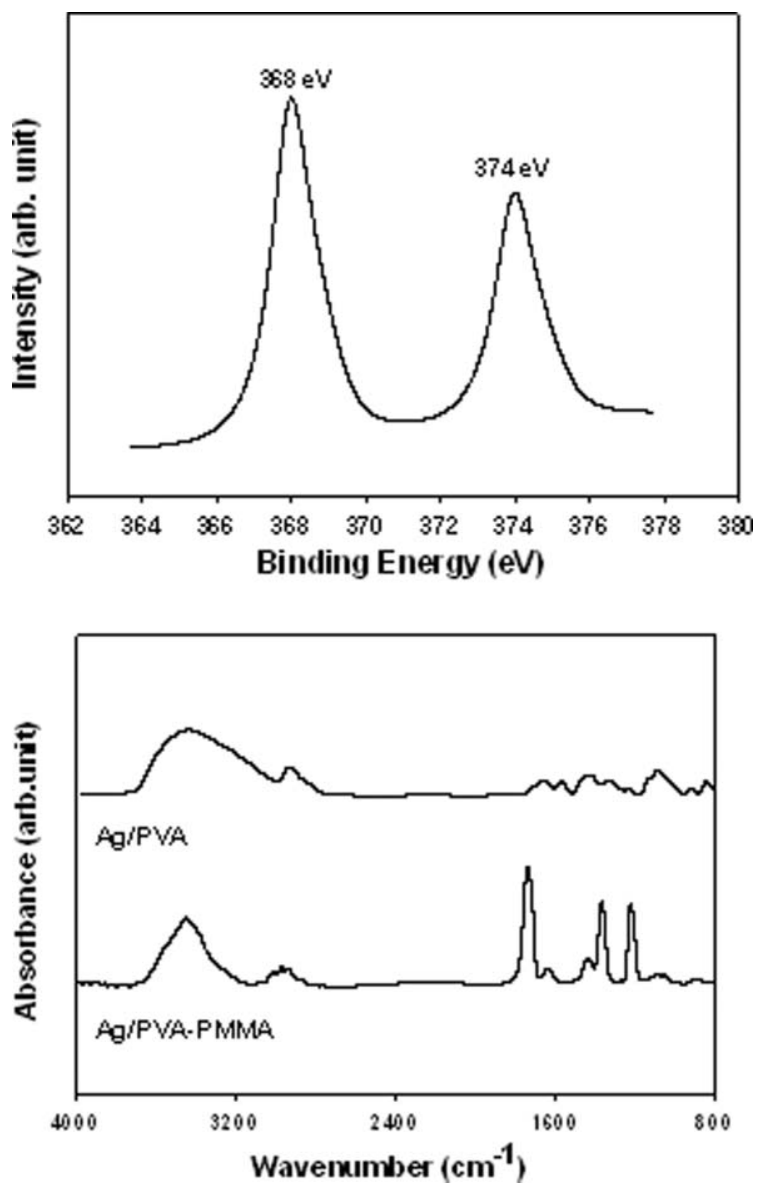

Fig. 2 (a) XPS and (b) FT-IR spectra of silver nanoparticle embedded polymer nanofibers. 

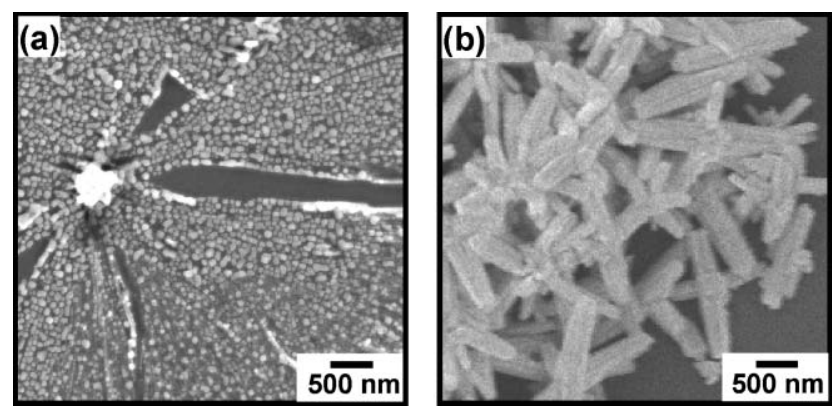

Fig. 3 SEM images of silver nanoparticle embedded polymer nanostructures (a) without monomer and (b) without PVA.

Table 1 Variation of nanoparticle size and nanofiber diameter with the silver ion/PVA ratio

\begin{tabular}{lll}
\hline $\begin{array}{l}\text { Weight ratio of } \\
\text { silver ion to PVA }\end{array}$ & $\begin{array}{l}\text { Average size of silver } \\
\text { nanoparticles/nm }\end{array}$ & $\begin{array}{l}\text { Average diameter of } \\
\text { polymer nanofibers/nm }\end{array}$ \\
\hline $4: 1$ & 8.0 & 30.2 \\
$2: 1$ & 7.4 & 27.4 \\
$1: 1$ & 6.7 & 13.2 \\
$1: 2$ & 6.5 & 10.1 \\
$1: 4$ & 6.4 & 9.7 \\
\hline
\end{tabular}

from the $\mathrm{O}-\mathrm{H}$ stretching vibration. Additionally, the characteristic FT-IR peaks of PMMA appeared at 1738 and $1220 \mathrm{~cm}^{-1}$ in the Ag-PVA-PMMA spectrum, due to the $\mathrm{C}=\mathrm{O}$ stretching and $\mathrm{C}-\mathrm{O}-\mathrm{C}$ stretching vibrations, respectively. This spectrum confirmed that polymerization had taken place successfully at the surface of the silver-PVA complexes.

In order to verify the generation of silver-polymer nanofibers during the polymerization, a blank system containing only silver ions and PVA (without monomer) was examined. There was no fiber formation from this silver-PVA system, as can be seen in Fig. 3a. Instead of fibers, only two-dimensional assemblies of silver nanoparticles were formed, with a "wheat sheaf" structure because of the crystallinity of PVA. ${ }^{16}$ From this experiment, it can be concluded that silver-embedded polymer nanofibers were fabricated during the radical-mediated dispersion polymerization. For comparison, Fig. $3 \mathrm{~b}$ shows the SEM image of silver nanoparticle embedded PMMA nanorods. The silver-PMMA system (without PVA) produced polymer nanorods with a small aspect ratio, and with larger silver nanoparticles than the silver-PVA-PMMA system. This control experiment indicated that PVA acts as a gelator to disperse and grow nanofibers in the axial direction, and as a stabilizer to protect the silver nanoparticles from aggregation.

To investigate in detail the effect of the amount of PVA, average diameters of silver nanoparticles and polymer nanofibers were analyzed while changing the weight ratio of silver ion to PVA (Table 1). The size and diameter were measured directly from each TEM image. In a each case, 100 silver nanoparticles and polymer nanofibers were counted, and the values averaged. As the ratio of silver ions to PVA increased, the size of the silver nanoparticles and the diameter of the polymer nanofibers both increased. When small amounts of PVA were used, larger silver nanoparticles and thicker polymer nanofibers are obtained because PVA cannot effectively stabilize the silver nanoparticles and the polymer nanofibers during polymerization. Since the polymer nanofibers had been grown from the assemblies of silver nanoparticles, the differences between the sizes of the polymer nanofibers were more significant than those of the silver nanoparticles. The role of PVA as a stabilizer and a gelator are therefore proved indirectly from this data.

In conclusion, the radical-mediated dispersion polymerization technique is found to be a facile one-step method for the fabrication of silver nanoparticle embedded polymer nanofibers in situ. The AIBN radical acted as a reductant and initiator during the polymerization process, and PVA played a role both in protecting the silver nanoparticles from aggregation and in producing the polymer nanofibers. This novel approach could be expanded to the synthesis of other metal nanoparticles and diverse polymer nanofibers.

This work was supported by the Brain Korea 21 program of the Korea Ministry of Education and the Korea Science and Engineering Foundation through the Hyperstructured Organic Materials Research Center.

\section{Notes and references}

1 Y. Li, Y. Wu and B. S. Ong, J. Am. Chem. Soc., 2005, 127, 3266; S. Matsuda, Y. Yasuda and S. Ando, Adv. Mater., 2005, 17, 2221; Y. Lu, G. L. Liu and L. P. Lee, Nano Lett., 2005, 5, 5; A. M., Z. Sun, K. Hindi, A. Milsted, D. Ely, D. H. Reneker, C. A. Tessier and W. J. Youngs, J. Am. Chem. Soc., 2005, 127, 2285; W. Caseri, Macromol. Rapid Commun., 2000, 21, 705.

2 G. Carotenuto, L. Nicolais, B. Mortorana and P. Perlo, Metal-Polymer Nanocomposites, John Wiley \& Sons, New Jersey, 2004, ch. 5.

3 M. H. Lim and D. G. Ast, Adv. Mater., 2001, 13, 718.

4 S. Porel, S. Singh, S. S. Harsha, D. N. Rao and T. P. Radhakrishnan, Chem. Mater., 2005, 17, 9; Z. Li, H. Huang, T. Shang, F. Yang, W. Zheng, C. Wang and S. K. Manohar, Nanotechnology, 2006, 17, 917; S. Rifai, C. A. Breen, D. J. Solis and T. M. Swager, Chem. Mater., 2006, 18, 21

5 X. Wang, K. Naka, H. Itoh, S. Park and Y. Chujo, Chem. Commun., 2002, 1300.

6 L.-B. Luo, S.-H. Yu, H.-S. Qian and T. Zhou, J. Am. Chem. Soc., 2005, 127, 2822; L.-B. Luo, S.-H. Yu, H.-S. Qian and J.-Y. Gong, Chem. Commun., 2006, 793.

7 A. Chen, K. Kamata, M. Nakagawa, T. Iyoda, H. Wang and X. Li, J. Phys. Chem. B, 2005, 109, 18283; A. Chen, H. Wang and X. Li, Chem. Commun., 2005, 1863.

8 C.-W. Chen, M.-Q. Chen, T. Serizawa and M. Akashi, Adv. Mater., 1998, 10, 1122; C.-W. Chen, T. Serizawa and M. Akashi, Langmuir, 1999, 15, 7998; C.-W. Chen, T. Serizawa and M. Akashi, Chem. Mater., 2002, 14, 2232.

9 Q. F. Wei, H. Ye, D. Y. Hou, H. B. Wang and W. D. Gao, J. Appl. Polym. Sci., 2005, 99, 2384; R. J. Tseng, J. Huang, J. Ouyang, R. B. Kaner and Y. Yang, Nano Lett., 2005, 5, 1077; J. Jang, Adv. Polym. Sci., 2006, 199, 189.

10 W.-J. Jin, H. K. Lee, E. H. Jeong, W. H. Park and J. H. Youk, Macromol. Rapid Commun., 2005, 26, 1903; Y. Wang, Q. Yang, G. Shan, C. Wang, J. Du, S. Wang, Y. Li, X. Chen, X. Jing and Y. Wei, Mater. Lett., 2005, 59, 3046.

11 R. G. Alargova, V. N. Paunov and D. V. Orlin, Langmuir, 2006, 22 765; R. G. Alargova, K. H. Bhatt, V. N. Paunov and O. D. Velev, Adv. Mater., 2004, 16, 1653; J. R. Fried, Polymer Science and Technology, Prentice-Hall, New Jersey, 1995, ch. 11, p. 387.

12 J. Liao, Y. Zhang, W. Yu, L. Xu, C. Ge, J. Liu and N. Gu, Colloids Surf., A, 2003, 223, 177.

13 M. Kimura, S. Kobayashi, T. Kuroda, K. Hanabusa and H. Shirai, Adv. Mater., 2004, 16, 335.

14 T. Huang and R. W. Murray, J. Phys. Chem. B, 2003, 107, 7434; P. C. Joanna, K. Khambaswadkar and B. R. Christopher, Ind. Eng. Chem. Res., 2000, 39, 4749.

15 X. Sun, S. Dong and E. Wang, Macromolecules, 2004, 37, 7105; X. Wang, J. C. Yu, C. Ho and A. C. Mak, Chem. Commun., 2005, 2262.

16 K. E. Strawhecker and E. Manias, Macromolecules, 2001, 34, 8475. 